\title{
Editor-in-Chief's Foreword
}

It is my honor and pleasure to present the tenth issue of Translocal Chinese: East Asian Perspectives, which also marks the fifth year of our partnership with Brill. Hence, it is an occasion of double jubilee! Moreover, I am delighted to announce that the journal has been included in the ERIH PLUS and Scopus reference indexes since May, 2020. This is evidence that the journal has lived up to its mission. We take pride in serving an important international readership and will continue to strive for further improvements in our impact and visibility. We take seriously our role in establishing a platform to enhance the understanding of overseas Chinese. This is even more urgent and important when we see the rise of Chinese nationalism on the one hand, and the reinforcement of anti-Chinese sentiment in the wake of Covid-19, on the other.

As such, we are pleased to present the current issue, which, under the invitation of executive editor, Dr. Leo Douw, includes a position statement by Dr. Wu Xiao An. Dr. Wu is Professor of History at Peking University and also the Director of the Center for the Study of Chinese Overseas at Peking University. We hope the publication of Dr. Wu's piece will stimulate further debates on the future of overseas Chinese studies. We thus welcome contributions in response to Dr. Wu's essay. Through these discussions, we expect to further hone the journal's mission and deepen its commitment to transnational and transdisciplinary studies of overseas Chinese and their communities. We intend to publish selected responses to Dr. Wu's statement in a special issue (15.1), which is scheduled to be published in May 2021. Please do not hesitate to contact me if you have any questions concerning the journal in general, or the contributions to the special issue.

\footnotetext{
Ming-Feng Liu

Associate Professor, Program of Culture and History of Hokkien, National Quemoy University, Kinmen, Taiwan; Editor-in-Chief of TCEA mf6o129@nqu.edu.tw
} 